Georg Kreis (Hrsg.)

Frieden und Sicherheit Israel und die Nahostkrise als europäisches Thema 
Das Europainstitut der Universität Basel ist ein rechts-, politik- und wirtschaftswissenschaftliches Zentrum für interdisziplinäre Lehre und Forschung zu europäischen Fragen. Neben einem einjährigen, praxisbezogenen und interdisziplinären Nachdiplomstudium zum Master of Advanced European Studies und dem Vertiefungsstudium Major in Conflict and Development werden spezielle Weiterbildungskurse angeboten. In der Forschung werden in Zusammenarbeit mit benachbarten Instituten sowohl fachspezifische wie multidisziplinäre Themen bearbeitet. Das Europainstitut ist als Ansprechpartner für Politik, Wirtschaft und Verwaltung beratend tätig.

\section{ISBN-13: 978-3-905751-03-1}

ISBN-10: 3-905751-03-8

Georg Kreis, 1943 in Basel geboren, Leiter des Europainstituts der Universität Basel.Ordinarius für Neuere Allgemeine Geschichte und Schweizergeschichte an der Universität Basel.

Ernest Goldberger, 1931 in Basel geboren, Inhaber eines Beratungsunternehmens für Exportfragen, lebt seit 1991 in Israel, Autor des Buches „Die Seele Israel. Ein Volk zwischen Traum, Wirklichkeit und Hoffnung“. Zürich NZZ-Verlag 2004, 492 S.

Arthur Hottinger, 1926 in Basel geboren, Korrespondent für NZZ und Schweizer Radio zunächst in Beiruth, dann in Madrid und Nikosia. Seit 1992 pensioniert, lebt heute in Madrid und Lausanne. Zahlreiche Publikationen. Zuletzt „Islamische Welt. Der Nahe Osten, Erfahrung, Begegnungen, Analysen. Zürich NZZ-Verlag 2004, 750 S.

Laurent Goetschel, 1965 in Bern geboren, Professor für Politikwissenschaft am Europainstitut der Universität Basel und Direktor der Schweizerischen Friedensstiftung (swisspeace), leitete das Nationale Forschungsprogramm "Schweizer Aussenpolitik“ (NFP 42) und war Berater von Bundesrätin Micheline Calmy-Rey während ihres ersten Jahres als Aussenministerin.

\section{Inhaltsverzeichnis}

\section{Einleitung}

von Georg Kreis

Doppelkrise im Nahen Osten:

Der Konflikt zwischen Israel und Palästina

von Ernest Goldberger

Der Terrorismuskrieg im Irak

von Arnold Hottinger

\section{Perspektiven des Nahostkonfliktes und die}

Rolle der Schweiz 


\section{EinLeitung}

\section{Israel-Irak}

von Georg Kreis

Zum Titel der Schrift: „Frieden und Sicherheit" sind die beiden zentralen, aber in einiger Ferne liegenden Ziele, um die es in der angesprochenen Problematik geht. Am liebsten hätte man beides gleichzeitig. Solle es aber nicht ohne Staffelung gehen, dann müsste man dem Frieden den Vorzug geben mit der Einsicht, dass es ohne Frieden keine Sicherheit gibt. Und wer meint, dass es Sicherheit ohne Frieden gibt, der beschert der Region höchst unsichere Verhältnisse. „Israel und die Nahostkrise als europäisches Thema" will zum Ausdruck bringen, dass es hier bei allem Respekt vor Eigenständigkeit um sehr europäische Themen geht, weil Europa im Schlechten wie im Guten in dieser Region stets mit von der Partie gewesen ist und schon darum auch heute eine verantwortungsvolle Haltung einnehmen muss.

Der Nahe Osten beschäftigt. Zwei der hier veröffentlichten Referate, diejenigen von Ernest Goldberger und Arthur Hottinger, wurden im November 2005 in der vollen Aula der Universität Basel vorgetragen. Beide sind wegen ihrer zeitlosen Qualität, aber auch wegen der - leider - unveränderten Problemlage auch heute noch hochaktuell. Dies gilt auch für den dritten, von Laurent Goetschel, dem Leiter des politikwissenschaftlichen Bereichs des Europainstituts, verfassten Text, der sogar noch ein Jahr älter ist, aber eine ausgezeichnete Ergänzung zu den beiden anderen und eine nach wie vor aktuelle Analyse ist. Arafat und Sharon sind nicht mehr, dagegen ist Hamas nun auch ein politischer Akteur geworden. Man kann darin eine Bestätigung der stets behaupteten Aussichtslosigkeit einer friedlichen Lösung, man kann darin aber auch ein Tor sehen, durch das der unverzichtbare Friedensprozess eben gehen muss. Jedenfalls sind die Grundzüge 
des Konflikts die gleichen geblieben.

„Hier sehe ich...", „Ich bin der Ansicht...", „Ich ziehe Bilanz..." - solche Formulierungen findet man zuhauf in den Texten, deren Vortragscharakter man bewusst gelassen hat. Damit behalten sie ihre im doppelten Sinn ansprechende Qualität und wird bekräftigt, dass engagierte Stellungnahmen vorliegen. Das bedeutet überhaupt nicht, dass hier willkürlich subjektive Meinungen verbreitet werden, sondern persönlich erarbeitete Befunde als Ergebnisse von sorgfältiger Auseinandersetzung mit der Sache.

Ernest Goldberger stellt sich als Beobachter und Betroffener. Zuweilen will es scheinen, dass der israelisch-palästinensische Konflikt ein symmetrischer sei. Dass der grösste Teil der kritischen Bemerkungen Israel betrifft, erklärt sich aus dem Umstand der biographischen Verbundenheit mit dieser Seite, aber doch auch aus der impliziten wie expliziten Meinung, dass der Schlüssel zur Lösung oder mindestens Entschärfung der Problematik auf dieser Seite liegt. So sehr inm dabei auch die palästinensische Seite ein Anliegen ist, den Irrweg, auf dem sich Israel nach seiner Meinung befindet, kritisiert Goldberger vor allem um Israel selbst, das heisst um dessen Seele Willen, wie der Titel von Goldbergers bekannter Schrift lautet. Der Irrweg besteht nach der Meinung des Verfassers darin, dass aus dem zionistischen Projekt bereits von den Gründungsvätern eine mehrheitlich nationalistische Gesellschaft und ein halbtheokratisches Staatswesen gemacht wurde. Das Wort fällt so nicht, aber die Ausführungen sind so gemeint: Israel sollte ein normaler Staat werden, mit einer Verfassung, ein säkularer Staat, in dem Religion und Staat getrennt sind.

Arthur Hottinger zeigt auf, dass die Regierung Bush den Krieg mit dem Irak gesucht und mit fadenscheinigen Begründungen gerechtfertigt hat; einen Krieg, der die Lage der irakischen Bevölkerung noch schlimmer als zu Saddams Zeiten gemacht hat und, gelinde gesagt, eine fatale Belastung für die ganze Region ist - mithin das Gegenteil von dem, was angestrebt oder wenigsten angegeben wurde. Hottinger macht auch auf die Parallelität und Verknüpfung zwischen den amerikanischen Neocons und dem rechten Flügel der rechten LikudPartei Israels aufmerksam. Ohne zu behaupten, dass Israel die amerikanische Intervention im Nahen Osten gewünscht hat, stellt Hottinger fest, dass Israel von der Dauerkrise im Irak profitiert. Allerdings, wie man Hottinger verstehen muss, zu seinem Nachteil profitiert. Denn sie gibt Spielraum für eine Weiterführung der ungerechten und unheilvollen Verdrängung der palästinensischen Bevölkerung.

Laurent Goetschel empfiehlt in seiner systematischen Politikanalyse eine nüchterne, aber nicht fatalistische Betrachtung. Zum besseren Verständnis soll man Konfliktualität als Normalität anerkennen. Ferner sei der Dialog - selbst mit dem Feind - unausweichlich und sei auf beiden Seiten die Konfliktlösung nicht in gewaltsamen Vorgehensweisen zu sehen. Anderseits dürfe man sich nicht der Illusion hingeben, dass der Unilaterlismus die Lösung bringe. Wichtig sei die Entwicklung von konstruktiven Zukunftsperspektiven. Diese sieht Goetschel nicht bei den religiösen Eiferern beider Lager, schon gar nicht bei den Siedlern und auch nicht in der Art, wie Auserwähltheitsvorstellungen geltend gemacht werden. Goetschel setzt auf die Kreativität des guten Willens und ortet bisher nicht oder wenig ausgeschöpfte Möglichkeiten. Von der Schweiz sagt er, dass sie bei der Entwicklung oder Stärkung von Perspektiven behilflich sein kann und dass sie als Advokatin des humanitären Völkerrechts im Konfliktgebiet eine wichtige Rolle spielen muss.

Goetschel hielt seinen Vortrag im Rahmen des Jewish College in der Stadtbibliothek Bern am 1. November 2004, Goldberger und Hottinger referierten am 7. November 2005 im Rahmen eines Diskussionsabends, der, initiiert von Bernhard A. Probst, von der StatistischVolkswirtschaftlichen Gesellschaft Basel, der Handelskammer beider Basel und dem Europainstitut gemeinsam veranstaltet wurde. Der Verfasser dieser Zeilen bemerkte einleitend zu diesem Abend: Die 
Universität ist kein politisches Schlachtfeld, sie ist aber auch nicht ein Ort, an dem grosse politische Fragen ausgeblendet werden sollen. Heute Abend haben zwei Autoren das Wort, die - jeder auf seine Weise - ihre authentischen Einsichten vermitteln und jedenfalls wissen, wovon sie reden. Schon deswegen vermitteln sie ernst zu nehmende Botschaften. Auf Grund ihrer Ausführungen können wir in Kombination mit den Einsichten, die wir bereits in uns tragen, zusätzliche Orientierung in einem schwer zu überblickenden und noch schwerer zu verstehenden Konfliktfeld gewinnen.

Basel, 20. März 2006 (am 3. Jahrestag des Angriffs auf den Irak)

\section{Doppelkrise im Nahen Osten: Der Konflikt zwischen Israel und Palästina}

\section{von Ernest Goldberger}

Als erstes möchte ich den Trägern und Organisatoren dieses Anlasses für die Einladung danken, heute zu Ihnen sprechen zu dürfen. Ich bin bewegt, nach über einem halben Jahrhundert als Referent in meine Alma Mater zurückzukehren, in der ich lernen durfte, und wo mir Edgar Salin, mein unvergessener Lehrer und Mentor, bis heute zum Vorbild wurde für kritisches und unbestechliches Forschen und Denken in interdisziplinären Zusammenhängen, für die Suche nach den tragenden geistigen Kräften hinter wirtschaftlichen und soziologischen Vorgängen.

Ganz in diesem Sinne, und damit bin ich schon beim Thema, verordnete er mir einen mindestens dreimonatigen Aufenthalt in Israel, um vor Ort die notwendigen Recherchen für meine Dissertation über die Interdependenz gesellschaftlicher und volkswirtschaftlicher Prozesse im damals erst wenige Jahre alten Staat Israel vorzunehmen. Auch er hätte schliesslich seinerzeit für seine Doktorarbeit über ein anderes Entwicklungsland drei Monate in Alaska verbringen müssen, und dies bei bitterster Kälte, die ich nicht zu befürchten brauche, wie er augenzwinkernd hinzufügte.

In späteren Jahren getraute ich mich lange nicht, in meine dann entstandene Schrift hineinzuschauen. Ich fürchtete mich, in dunkle Abgründe jugendlicher Unerfahrenheit und Fehlprognosen blicken zu müssen. Erst auf diesen Abend hin habe ich mich dazu überwinden können und festgestellt, dass mir schon damals in der israelischen Gesellschaft gewisse psychosoziale Muster aufgefallen waren, die sich dann später massiv verstärkt haben, so durch die psychologischen Folgen von Israels fünf Kriegen, die Masseneinwanderung, die nukleare Option oder die Intifada. Die schicksalhafte Bedeutung die- 
ser Erscheinungen, die ich in den Ansätzen beobachtet hatte, ist mir aber erst bewusst geworden, als es mich vor 14 Jahren nach Israel verschlug, wo ich seither wohne.

Ich war nun nicht mehr bloss Beobachter, sondern Betroffener. Dadurch änderte sich ganz grundlegend der innere Bezug zu den Geschehnissen und deren Triebkräften. Der Prozess zur Bereitschaft einer unvoreingenommenen Realitätswahrnehmung ging zuerst zögernd vor sich, weil sich die menschliche Natur intuitiv gegen Desillusionierungen wehrt. Ausserdem gab es bis zur Ermordung von Ministerpräsident Rabin durch einen religiösen Nationalisten vor fast genau zehn Jahren echte Friedenshoffnungen. Danach brachen aber die Dämme des inneren Widerstandes gegen die Erkenntnis von bedrohlichen Erscheinungen umso rascher ein.

Die nicht mehr verdrängbaren Gefühle von Sorge und Unruhe schufen den Willen zu versuchen, diesen Erscheinungen unvoreingenommen auf den Grund zu gehen, die einstürmenden Gedanken zu hinterfragen und zu ordnen. So entstand mein Buch „Die Seele Israels".

Vier Jahre hatte ich an diesem Buch gearbeitet, doch heute fällt mir die sehr viel schwierigere Aufgabe zu, zu versuchen, Ihnen in 20 Minuten die Hintergründe des "Nahostkonfliktes“ aus israelischer Sicht zu umschreiben.

Ich werde auf drei Themenkreise eingehen: Was versteht man unter der Nahostkrise, soweit Israel involviert ist? Welches ist ihre globale Bedeutung und - vor allem: Was ist eigentlich ihr Kern, ihr Wesen?

Gemeinhin versteht man unter der sehr ungenauen, ja eigentlichen falschen Bezeichnung „Nahostkrise“ den Konflikt zwischen dem jüdischen Volk im Staat Israel und dem palästinensischen Volk, den zwei Völkern, die auf dem gleichen Flecken Erde leben und dort weiter leben wollen. Die Vertreter der jüdischen Bevölkerung Israels, welche mit etwa $70 \%$ wohlverstanden nur eine Mehrheit der 7 Millionen
Einwohner bilden und die Regierungsgewalt besitzen, machen historische und religiöse Rechte geltend, während die Palästinenser auf ihre natürlichen Rechte hinweisen, seit mindestens 13 Jahrhunderten dort ansässig zu sein. Israel fordert die vorbehaltlose und internalisierte Anerkennung seiner staatlichen Existenz durch die Palästinenser. Die schwerwiegende Problematik dieses Anspruchs liegt heute darin, dass die Frage der Grenzen offen ist, denn Israel hat im so genannten Sechstagekrieg vom Juni 1967 das Westjordanland mit OstJerusalem erobert und, entgegen der 4. Genfer Konvention von 1949, mit bisher rund 250‘000 jüdischen Siedlern bevölkert. Annektiert wurden nur Ost-Jerusalem und Gebiete um Jerusalem herum, wo heute weitere 200‘000 Juden leben, sodass Israel heute das einzige Land ohne feste Grenzen ist. Auch wenn der Abzug aus dem Gazastreifen im August dieses Jahres den Mythos der Irreversibilität von „facts on the ground" relativiert hat, setzen die jetzigen Machthaber auf die Kraft vollendeter Tatsachen, erweitern die Siedlungen und bauen die so genannte Schutzmauer weiter, nicht etwa auf der Grenzlinie von 1967, sondern teilweise weit im Inneren des Westjordanlandes. Ich fürchte, dass Israels Machthaber eines Tages diese Mauer unilateral zur Grenze erklären und so den Konflikt verewigen werden. Seit die etwa 7‘500 Siedler im August dieses Jahres aus dem Gazastreifen, einem Gebiet von einem Prozent des historischen Palästinas, abgezogen sind, hat sich die Zahl der Siedler im Westjordanland um 13‘000 erhöht. Dies bestärkt den Verdacht einer solchen Strategie.

Die Palästinenser fordern demgegenüber einen vollwertigen, lebensfähigen Staat auf den Gebieten des Gazastreifens und des Westjordanlandes, wobei die grundsätzliche Bereitschaft besteht, gewisse jüdische Siedlungsblöcke im Austausch gegen unbevölkerte Gebiete im Verhältnis eins zu eins an Israel abzutreten.

Bei rationaler Einschätzung bilden der Status von Jerusalem, das heute schon de facto eine geteilte Stadt ist, und die Frage der heiligen Stätten keine echten Hindernisse einer Einigung. Bedeutend 
schwerer belastet aber die Flüchtlingsfrage. Im so genannten Unabhängigkeitskrieg von 1948/49 flüchteten rund 700‘000 Palästinenser nach Jordanien, dem Gazastreifen, dem Westjordanland, Libanon und Syrien. Mindestens teilweise wurden sie vertrieben. Ihre Zahl ist heute in der bereits 4 . Generation auf 3 bis 4 Millionen angewachsen. Eine unbeschränkte Rückkehr dieser zum Grossteil in unwürdigen Verhältnissen lebenden Menschen würde das Ende des Staates Israel bedeuten, doch es gibt realistische Lösungskonzepte, allerdings keine ohne die Bereitschaft Israels, das psychologische Element zu beachten, also Verantwortung zu übernehmen und an einer Bewältigung dieser menschlichen Tragödie massgeblich beizutragen.

Mit Ägypten und Jordanien bestehen funktionierende Friedensverträge, mit dem nördlichen Nachbarstaat Libanon gibt es keine territorialen Fragen und mit Syrien müsste lediglich ein Kompromiss in der Frage der im Sechstagekrieg vom Juni 1967 eroberten und dann annektierten Golanhöhen gefunden werden. Würde Israel die greifbare Option einer Konfliktbeilegung mit den Palästinensern und den Nachbarstaaten wählen, werden alle islamischen Länder - die Saudis haben dies letztes Jahr unmissverständlich zu verstehen gegeben - ihre Beziehungen mit Israel normalisieren, vielleicht mit Ausnahme des Iran, der sich aber damit isolieren würde.

Bei der Frage der globalen Bedeutung dieses Konfliktes kommen historische Reminiszenzen auf: Wie vor bald 2000 Jahren beeinflusst ein kleines Volk auf einem winzigen Stück Erde die Geschicke der Menschheit mit einem Gewicht, das in einem geradezu surrealen Verhältnis zu seiner zahlenmässigen Grösse steht. Nur so wird verständlich, dass im August dieses Jahres 2000 Journalisten aus aller Welt in den Gazastreifen reisten, um die zynische Inszenierung der Freilichtaufführung des Abzuges von 1500 jüdischen Familien zu beobachten. Besonders unerträglich waren dabei die Szenenbilder, in denen Kinder, Holocaustopfer und religiöse Insignien missbraucht wurden. Dabei war alles bloss ein taktischer Schachzug zur Verstär- kung des Griffes auf das Westjordanland, unter Aufrechterhaltung der Absperrung des Gaza-Streifens zu Land, Wasser und Luft.

Die schwelende Krise belastet das weltpolitische und weltwirtschaftliche Klima schwer. Der islamische Terrorismus schöpft zwar seine Motivation nicht direkt aus dem Leid der Palästinenser, doch wird dieses oft zur Rechtfertigung mörderischer Taten vorgeschoben und Israel als Verkörperung der zu bekämpfenden westlichen Unkultur hingestellt. Mit der Unterlassung einer aktiven Friedenspolitik wird deshalb auch die Möglichkeit verpasst, dem Terrorismus und der Tendenz zur Entwicklung von Massenvernichtungswaffen endlich auch mit geistigen, und nicht wie bisher nur mit militärischen Kräften entgegen zutreten.

Vor allem aber gräbt sich die Rechtlosigkeit und menschenunwürdige Existenz der Palästinenser immer schmerzhafter in das Bewusstsein und Gewissen der zivilisierten Welt ein, wohlverstanden auch in das vieler Juden in- und ausserhalb Israels. Im 21. Jahrhundert gibt es keinen Raum mehr für Unterdrückung eines Volkes durch ein anderes, für das alte kolonialistische Prinzip „divide and rule“ oder für unilaterale Massnahmen, die Formen von Apartheid zur Folge haben. Die moralische Autorität der Vereinigten Staaten als einzige Weltmacht erleidet zudem durch die Tatsache Schaden, dass ihre Regierung die Möglichkeiten eines Druckes auf Israel nicht wahrnimmt und so zur Mittäterin wird.

Doch nicht nur diese Perspektiven verpflichten zum Nachdenken über das Wesen dieser Krise, sondern vor allem auch das Wissen der Historiker um die Vergänglichkeit aller Machtgebilde und die quälenden Fragen, wie Israel einen ewigen Krieg mit den Arabern mit seinen verderblichen Auswirkungen auf seine Gesellschaft überleben kann und das zionistische Experiment durch ein endloses Blutvergiessen nicht seinen Sinn verlieren könnte. Ich komme dabei immer wieder zur Erkenntnis, dass der israelisch-palästinensische Konflikt heute wesentlich als eine Manifestation einer tiefen inneren Krise Israels 
verstanden werden muss.

Für diese Ansicht schulde ich Ihnen natürlich eine Erklärung. Ich möchte zu diesem Zwecke zum zweiten Mal mehr als ein halbes Jahrhundert zurückblenden, an diesen Ort, ja in diese Aula, in der es mir vergönnt war, als junger Student einem Vortrag des Religionsphilosophen und Soziologen Martin Buber beizuwohnen. Einige der Ausführungen und Diskussionsvoten dieser beeindruckenden Persönlichkeit klingen mir bis heute nach. Buber war engagierter Zionist und Mitbegründer der 1925 in Jerusalem eröffneten Hebräischen Universität, wo er nach seiner Flucht aus Deutschland von 1938 bis 1951 Soziologie lehrte. Aber er war vor allem ein bedeutender Humanist, ein Verkünder universeller Werte, beispielsweise in seiner Philosophie der Ich-Du-Beziehung. Wie er, wollten Theodor Herzl, der als Gründer des politischen Zionismus angesehen wird, sowie die meisten anderen zionistischen Vorkämpfer den für sie sonst nicht besiegbaren Antisemitismus durch die Errichtung einer von der Welt anerkannten, sicheren Heimstätte für die Juden überwinden. Es ging innen aber dabei mindestens gleichermassen um die Schaffung eines produktiven neuen jüdischen Menschenbildes und einer gerechten Gesellschaft. Die Erreichung der zionistischen Ziele auf Kosten eines anderen Volkes war für diese Menschen undenkbar. Dies gilt auch für die bedeutenden Persönlichkeiten, welche mit dem Zionismus sympathisierten. Zu diesen gehörte beispielsweise Albert Einstein, der schon 1948 eindrücklich vor der Gefahr nationalistischer Strömungen in Israel warnte, aber auch mein schon erwähnter Lehrer Edgar Salin, der Israel in den Jahren 1958 bis 1969 oft besuchte und Initiator und spiritus rector von ökonomisch-soziologischen Forschungsprojekten über Israel der List-Gesellschaft war. Er mahnte schon damals mit seinem bewundernswert visionären Blick die Lösung des palästinensischen Flüchtlingsproblems als absolut vordringlich an, leider ohne genügend Gehör zu finden.

Ich kämpfe daher mit Überzeugung gegen das vielerorts verbrei- tete Klischee, der Zionismus sei ein Kind des europäischen Nationalismus. Ich rücke inn vielmehr in die Nähe der Aufklärung und des universellen Wertesystems der jüdischen Religion. Dieses hat sich schon gegen den entleerenden Kult im ersten und zweiten Tempel gestellt - ich erinnere in diesem Zusammenhang an die alten Propheten und vor allem an den Juden Jesus - und dann Eingang gefunden in die Exilreligion einer machtlosen, oft diskriminierten und verfolgten Minderheitsgruppe. Werte wie Nächstenliebe, Gerechtigkeit, Frieden, Achtung des Fremden und Schwachen und vor allem des menschlichen Lebens standen im Vordergrund.

Nur so ist es zu erklären, dass alle bemerkenswerten Errungenschaften des Zionismus ausnahmslos nicht nationalistisch motiviert waren. Dies gilt beispielsweise für die Kibbuz-Bewegung mit ihrem Streben nach individueller und sozialer Selbstverwirklichung und ihrer neuen Gesellschafts- und Eigentumsform, für die Gründung von weltoffenen Hoch- und Kunstschulen oder für das Weltgeltung besitzende Weizmann-Institute for Science.

Nach der Ausrufung des Staates Israel im Mai 1948 gerieten diese humanistisch-universellen Werte zunehmend in Widerstreit mit nationalistischen und pseudoreligiösen Strömungen und wurden von diesen immer mehr zurückgedrängt. Die psychologischen und soziologischen Triebkräfte dieser gefährlichen Entwicklung sind bisher zu wenig untersucht worden und die Darlegung meiner Sichten zu diesem Thema würde den Rahmen dieses Vortrages weit sprengen. Ich erwähne daher nur ein Stichwort von vielen möglichen: die tragische Unfähigkeit zur Loslösung von einer endlos kultivierten und instrumentalisierten historischen Opferrolle und der damit rationalisierte Fehlglaube, mit Hilfe der neu erworbenen Macht seien jetzt alle Konflikte lösbar.

Man muss in diesem Zusammenhang aus heutiger Sicht die Tatsache als schicksalsschwere historische Fehlleistung einstufen, dass es die verantwortlichen Gründer Israels, allen voran der erste Minis- 
terpräsident Ben Gurion, verfehlten, sich der Frage des geeigneten Staatscharakters zu stellen und eine solche Identität deutlich nach Innen und Aussen zu deklarieren. Ben Gurion wollte diesen Fehler mit dem Auftrag an die religiöse Orthodoxie gut machen, dem Staate einen „jüdischen Charakter" zu verleihen. So geriet er aber noch mehr auf Abwege, denn der so beschworene ,jüdische Charakter" ist unbestimmbar und daher eine Fiktion. Zudem überhäufte er zu diesem Zwecke die religiösen Machthaber mit Privilegien, Zugeständnissen und Monopolen und übertrug ihnen Aufgaben, die heute in jedem modernen Land Sache des Staates sind, und dies entgegen den ausdrücklichen und visionären Warnungen von Theodor Herzl in dessen Manifest "Der Judenstaat" aus dem Jahre 1896. So legte Ben Gurion als Atheist den Grundstein für eine kuriose Halbtheokratie und trug zur Entleerung der jüdischen Religion in Israel bei, denn Machtstreben zerstört allemal wahre Religiosität. Die Exponenten der Orthodoxie gehören zu den kompromisslosesten Gegnern einer Verständigung mit den Palästinensern auf dem Wege zu einem Frieden, fallen durch Beziehungslosigkeit zu humanistischen Werten und Realitäten auf und machten ausgerechnet Israel zum einzigen Land der westlichen Welt, in dem Juden keine Religionsfreiheit geniessen. Religiöser Zwang ist Machtausübung und zerstört daher die religiöse Substanz.

Auch wenn die Vertreter der nach der Staatsgründung dominierenden Arbeiter-Partei ebenfalls, wenn auch moderater, vom Geist des aufkommenden Nationalismus infiziert wurden, war dieser in seiner unerbittlichsten Form vor allem in der Cherut-Partei zu finden. Diese war eine Inkarnation der so genannten Bewegung der RevisionistenZionisten, die einen Jüdischen Staat beidseits des Jordans anstrebten. 1977 kam sie erstmals an die Macht und diktiert heute mit ihrer Nachfolge-Partei Likud die Politik Israels.

Nach dem Sechstage-Krieg vom Juni 1967 wurden Nationalismus und Macht durchdrungene „Religiosität" durch einen Messianismus zusätzlich aufgeladen und zur Triebkraft der Besiedlung der besetzten palästinensischen Gebiete, und so zum Haupthindernis für eine Befriedung der Region.

Die Werte eines universellen Humanismus, wie sie Martin Buber und andere zionistischen Vorkämpfer vertraten, und eine aufrechte und erdverbundene Alltagsreligiosität, wie sie vom Milchmann Tewje im Musical „Anatewka“ zur Erbauung von Millionen von Zuschauern in aller Welt vorgeführt wurde, sind grundlegend unvereinbar mit nationalistischen und religiösen Machtansprüchen. Der Widerstreit dieser Strömungen droht die israelische Gesellschaft innerlich zu zerreissen. Die Ermordung des den Frieden suchenden Ministerpräsidenten Rabin vor genau zehn Jahren ist nur eine besonders tragische und spektakuläre Manifestation dieser inneren Verfeindung, in der die Austragung von Auseinandersetzungen mit Gewalt zur Norm zu werden droht. Viele Politiker zeigen sich daher nur mit Leibwächtern in der Öffentlichkeit, geniessen dies aber als ein Statussymbol und Beweis ihrer angeblichen Bedeutung. Wenn unser Ministerpräsident Lust auf einen kleinen Ausgang hat, so wird er von einer ganzen Schar Männern mit finsterer Miene begleitet, was die Steuerzahler Millionen kostet. Wenn ich bei solchen Fernsehbildern dann bemerke, in der Schweiz fahre der Bundesrat ohne Begleitung mit der Tram in sein Büro im Bundeshaus, und darin symbolisiert sich die Bedeutung der Gemeinsamkeit von bestimmten Werten bei allen Verschiedenheiten, hält man mich für einen Märchenerzähler.

Die Desintegration der israelischen Gesellschaft in sich teilweise feindlich gesinnte und dialogunfähige Gruppen und Untergruppen ohne ein solches Band gemeinsamer Werte, ja ohne Anerkennung der geltenden Gesetze als übergeordnetes Prinzip, verhindert eine rationale Politik und entzieht einem grossen Teil der Bevölkerung die Motivation zur aktiven politischen Mitwirkung. So konnte sich jene charakteristische schweigende Mehrheit herausbilden, wie sie von Historikern und Soziologen auch für andere Länder und Zeitphasen 
analysiert worden ist. Resigniert wird das Feld Minderheiten überlassen, welche die Richtung bestimmen vermöge ihrer ideologisch aufgeladenen Aggressivität, ihren Drohungen, Erpressungen und Verbreitung von Angst. Sie verfügen zudem über reichlich finanzielle Mittel und werden unterstützt von Politikern, die auf der Suche nach Befriedigung persönlicher Ambitionen um die Gunst dieser lautstarken Gruppen buhlen. Als Herr Netanyahu seinen Feldzug gegen Ministerpräsident Sharon um die Führung des Landes ankündigte, trat er als erstes in der Siedlerstadt Ma'ale Adumin auf und wandte sich nicht etwa an die Massen in der Konglomeration Tel Aviv. So können extremistische Siedler, orthodoxe Rabbiner und Ultra-Nationalisten eine aktive Friedenspolitik unterbinden. Zudem belasten sie die Gesellschaft mit gravierenden Mängeln und Fehlstrukturen, angefangen vom Fehlen einer Verfassung, die Nichttrennung von Staat und Religion, dem Niedergang des Erziehungssystems und der Lenkung von Staatsausgaben auf unproduktive Zwecke bis hin zur Kürzung der Sommerzeit.

Gewiss ist der Nahost-Konflikt auch eine Manifestation einer inneren Krise in der palästinensischen Gesellschaft, wie immer diese entstanden und durch die Politik Israels beeinflusst worden ist. Ich bin jedoch nicht befugt, diese als Aussenstehender zu analysieren. Auch als unvergleichlich schwächere Konfliktpartei steht den Palästinensern eine aussichtsreiche Friedensstrategie offen: gänzlicher Verzicht auf Terror zugunsten eines organisierten passiven Widerstandes, Anerkennung der Realität der Existenz des Staates Israel in den Grenzen von 1967 mit der Option eines Gebietsabtausches, Bereitschaft zu einer realitätsbezogenen Lösung der Flüchtlingsfrage mit Hilfe Israels und des Westens, Intensivierung von persönlichen Kontakten zu Israelis und Unterstützung des israelischen Friedenslagers, Aufbau eines effizienten Verwaltungssystems mit möglichster Ausschaltung der Korruption und Entwicklung eines klaren Konzeptes für den Aufbau situationsgerechter Wirtschaftsstrukturen zur Verminderung der Arbeitslosigkeit und der ökonomischen Abhängigkeit von Israel. Die Palästinenser könnten so über Nacht mit grosszügiger weltweiter Unterstützung rechnen.

Ich ziehe Bilanz: Der so genannte Israelisch-Palästinensische Konflikt ist heute letztlich ein Kampf zwischen den friedensfähigen Kräften in beiden Lagern gegen die friedensunfähigen Kräften in beiden Lagern. Diese Friedensfähigkeit ist eng verbunden mit dem Willen und der Erziehung zum gegenseitigen Verstehen. Dies ist nur möglich, wenn auf beiden Seiten die Geschichtsschreibung Mythen und Lügen über Bord zu werfen bereit ist und so durch eine Annäherung der Geschichtsbilder den Weg bereitet für Versöhnung und Verzeihung. "There is no future without foregiveness" lautet der Titel eines Buches des südafrikanischen Bischofs und Friedens-Nobelpreisträgers Desmond Tutu. Ohne eine solche Geisteshaltung wird das grossartige Potenzial dieser Region weiterhin brach liegen und die Hoffnung auf Frieden und menschenwürdiges Leben, welche die Mehrheit beider Völker hegt, unerfüllt bleiben. Wir sind Zeitzeugen einer Manifestation der ewigen Auseinendersetzung in der Menschheitsgeschichte zwischen geistigem Wachstum und Destruktivität, wenn Sie wollen zwischen Gut und Böse.

Der Ausgang dieses Kampfes wird bestimmen, was es mit der endlos besungenen Heiligkeit dieses geschundenen Landstriches in Tat und Wahrheit auf sich hat. 


\section{Der Terrorismuskrieg im Irak}

von Arnold Hottiger

Wir wissen heute, dass die Bush Regierung den Krieg gegen Saddam Hussein schon vor dem 23. Juni 2002 plante. Mindestens 8 Monate bevor er dann ausgelöst wurde. Kriegsgründe, die dem amerikanischen Publikum einleuchten würden, gab es keine. Deshalb mussten sie erfunden werden. In einem später bekannt gewordenen geheimen Memorandum, bekannt als das Downing Street Memorandum (zuerst veröffentlicht in der Sunday Times vom 1. Mai 05) erklärte das Oberhaupt des britischen Geheimdienstes nach Besuch in Washington am 23. Juni 2002 seinem Ministerpräsidenten Tony Blair, der Krieg sei beschlossene Sache.

«C [Dearlove] reported on his recent talks in Washington. There was a perceptible shift in attitude. Military action was now seen as inevitable. Bush wanted to remove Saddam, through military action, justified by the conjunction of terrorism and WMD. But the intelligence and facts were being fixed around the policy.» ${ }^{1}$

Es gab bekanntlich weder WMDs (weapons of mass destruction) noch eine Zusammenarbeit mit al-Qaida im Irak. Beides waren lügenhafte Propagandabehauptungen, dazu bestimmt, die Zustimmung des amerikanischen Parlamentes und der Bevölkerung zu erschleichen. „Informationen und Tatsachen seien um den politischen Entscheid herum zu fixieren", wie man den englischen Text auch übersetzen kann.

Dieser Sachverhalt wirft die Frage auf: Was wollte Bush mit seinem Krieg im Irak eigentlich erreichen? Gewiss, Saddam absetzen. Doch warum? Die einzige Antwort, welche die amerikanischen Verantwortlichen auf diese Frage bisher gegeben haben, nachdem klar geworden war, dass die gegebenen Kriegsbegründungen blosse Vorwände 
waren, ist: Sie wollten im Irak ein demokratisches Regime einrichten. Wer das Land kennt, musste wissen, dass es wahrscheinlich leichter sein würde, ein demokratisches Regime auf dem Mond zu errichten als im Irak. Vermutlich hätten sowohl die Bevölkerung wie auch die Parlamentarier der USA unbequeme Fragen gestellt, wenn die Bush Regierung von Beginn an zugegeben hätte, was sie heute offen erklärt: Demokratie sei das Ziel ihrer Kriegshandlungen im Irak. Von den Fragen der Legalität eines Krieges mit derartigen Zielen ganz abgesehen.

Es lässt sich einwandfrei dokumentieren (am einfachsten über die Web Site Project for the New American Century), dass die einflussreiche Gruppe innerhalb der amerikanischen Regierung, die man zusammenfassend als "Neocons" bezeichnet, die Idee eines Krieges gegen den Irak mit dem Ziel, das Land zu demokratisieren, seit mindestens 1997 verfolgte. Die Neocons zogen schon damals und ziehen weiterhin gerne die falsche Parallele: Amerika habe Nazi-Deutschland und Japan besiegt, beide seien daraufhin vorbildliche Demokratien geworden. Warum, fragten sie, solle dies im Irak nicht ebenso gehen. Einflussreiche Mitglieder der Verwaltung wie Paul Wolfowitz, Richard Bolton, Lewis Libby, Douglas Firth sowie innen nahestehende Minister wie Donald Rumsfeld und Vizepräsident Dick Cheyney vertraten diese Idee seit Jahren und warben für sie in ihren Publikationen und Reden. Bush liess sich offensichtlich von ihnen leiten, wenn nicht gar manipulieren.

Die Demokratie, die sie sich von dem Krieg gegen den Irak versprachen, sollte eine „kapitalistische Demokratie nach amerikanischem Vorbild werden“. Sie glaubten, diese Demokratie werde dann auf den Rest der arabischen und vielleicht gar muslimischen Staaten „ausstrahlen" und so das politische Bild des Nahen Ostens radikal verändern, gewissermassen „umpolen“. Zu einer solchen Veränderung werde auch gehören, erklärten sie, dass die neuen Musterdemokratien, Israel lieben und schätzen lernten.
Einige der Neocons (Richard Perle als Leiter der Gruppe, Douglas Feith, Charles Fairbank, David Wurmser u.a), die sich Study group on a new Israeli policy towards 2000 nannten, hatten im Rahmen des „Institute for Advanced Strategic and Political Studies“ Benjamin Netanyahu auf die Wahlen des Jahres 2000 hin neue politische Ideen nahegelegt, die sie A Clean Break, a new Strategy for Securing the Realm betitelten. Also etwa: „Ein sauberer Neuanfang, neue Strategie für Sicherstellung des Herrschaftsbereiches“. Mit dem Herrschaftsbereich ist das gemeint, was andere Grossisrael nennen. Das Institut ist in Jerusalem gelegen und besitzt ein Zweiginstitut in Washington. Es gilt als ein „Think Tank“ für Likud.

Die Gruppe empfahl „peace for peace" statt „land for peace“ in Palästina. Über den Irak sagt der Bericht:

"Israel can shape its strategic environment, in cooperation with Turkey and Jordan, by weakening, containing, and even rolling back Syria. This effort can focus on removing Saddam Hussein from power in Iraq - an important Israeli strategic objective in its own right - as a means of foiling Syria's regional ambitions. Jordan has challenged Syria's regional ambitions recently by suggesting the restoration of the Hashemites in Iraq.... ${ }^{2}$

Es gibt zahlreiche andere Indizien für die Nähe der Neocons zur israelischen Likud Partei und ihrer Politik. Ihre Besessenheit bezüglich eines Irak-Kriegs hat man sich wohl primär durch ihre Zusammenarbeit mit der israelischen Likud Partei zu erklären. Bei den Neocons wie bei anderen amerikanischen Politikern mag sich auch das irakische Erdöl auf den Kriegsentschluss ausgewirkt haben. Bush persönlich war nach dem Terrorschlag von New York und Washington offensichtlich von der Rolle fasziniert, die er als oberster Kriegskommandant im „Krieg gegen den Terrorismus“ zu spielen vermochte. Die Kriegsziele und Kiegsmethoden in diesem angeblichen Krieg waren sehr vage geblieben, und die Neocons profitierten offensichtlich von dieser 
Lage, um dem Präsidenten ihren seit Jahren gehegten Plan eines Krieges gegen den Irak nahezulegen, wenn nicht unterzuschieben.

Die Zahl der Opfer in dem irakischen Terrorkrieg nimmt immer noch weiter zu: Nach den Angaben des Pentagons betrug sie seit Januar 2004 (bis Ende August 2005) 26000 Iraker, zu denen noch die seit dem offiziellen Ende des Krieges gefallenen 2000 amerikanischen Soldaten kommen. In den ersten Monaten des Jahres 2004 betrug die Durchschnittszahl der irakischen Opfer täglich 26. Sie war bis August 2005 auf 64 im Tag angestiegen. (NZZ, 1. Nov. 2005 „Ungebrochene Gewalt im Irak").

Die militärischen Fachleute unterstreichen seit langem, dass die Amerikaner nicht über genügend Soldaten verfügen, um das ganze Land zu befrieden. Die Regierung in Washington wollte dies zuerst nicht wahr haben, gab es jedoch in der jüngsten Zeit indirekt zu, indem sie erklärte, die Iraker müssten selbst für die Sicherheit in ihrem Land sorgen. Zu diesem Zweck würden Truppen und Polizeikräfte ausgebildet. Doch gibt es nur wenige irakische Einheiten, die einsatzbereit sind, und kaum eine, die ohne amerikanische Unterstützung in den Kampf ziehen kann. Fachleute berichten, die irakischen Einheiten seien oft von den Guerrillas infiltriert; es komme auch vor, dass sie einfach nicht erschienen, wenn sie eingesetzt werden sollten.

Die oben genannten Opferzahlen sind gewiss Minimalzahlen, weil sehr oft unklar ist, wie weit die Opfer krimineller Mordtaten miteingerechnet, ja wie weit sie den Amerikanern auch nur bekannt sind. Diese Untaten spielen sich im Schatten der allgemeinen Unsicherheit $\mathrm{ab}$, die durch den undurchdacht und schlecht geführten amerikanischen Krieg verursacht wurde und die bis heute eher zunehmend als abnehmend andauert.

Die irakische Zivilbevölkerung ist offenbar ziemlich einstimmig der Meinung, ihre Lage sei gegenwärtig bedeutend schlechter als unter Saddam. Damals, so werden die Iraker immer wieder zitiert, herrschte immerhin Sicherheit für alle jene, die sich nicht an der Politik be- teiligten und über den tyrannischen Machthaber schwiegen. Heute müssen alle Reichen und Angehörigen des Mittelstandes befürchten, dass ihre Kinder und Frauen entführt werden, um Lösegeld zu erpressen. Sie werden mit dem Tod bedroht, wenn ihre Angehörigen nicht bezahlen. Dies ist der Hauptgrund für die Flucht der Eliten und Reichen in die arabischen Nachbarländer und ins weitere Ausland. Besonders die Flucht der Ärzte wird oft erwähnt. Wohl weil die Zurückgebliebenen sie am meisten vermissen.

Um nicht erschossen zu werden, lebt die irakische Regierung mit den Amerikanern in dem befestigten Stadtteil hinter der Grünen Linie. Was natürlich den Verdacht der Bevölkerung steigert, sie bestehe einzig aus Marionetten der Besetzungsmacht.

Die amerikanischen Truppen, die auf Patrouille ausgeschickt werden, zeigen sich immer brutaler. Die Angst veranlasst sie, „zuerst zu schiessen, dann Fragen zu stellen“. Dies führt zur Tötung zahlreicher unschuldiger Iraker. Oftmals fühlen sich ihre Verwandten verpflichtet, für ihre Angehörigen Rache zu nehmen. Die Stammestraditionen sind noch soweit lebendig, dass dies in weiten Kreisen als eine Ehrensache der Angehörigen und ihrer Stammesgenossen gilt. Wer sich rächen will, stösst zu den Guerrilleros des „Terrorismus“.

Die Guerrillas scheinen darauf auszugehen, einen Bürgerkrieg zwischen den irakischen Sunniten und den Schiiten zu provozieren, indem sie Schiiten töten. Die schiitischen Geistlichen haben ihre Gläubigen aufgerufen, nicht zu Rachemorden zu greifen. Dennoch ist es in den jüngsten Wochen zunehmend zu solchen gekommen. Die Spannungen zwischen den beiden Gemeinschaften wachsen offensichtlich an. Bisher hat der einflussreichste aller schiitischen Geistlichen, Ayatollah Ali Sistani, seine Gläubigen aufgefordert, mit den Besetzungsmächten zusammenzuarbeiten. Doch seine Mitarbeiter haben kürzlich erklärt, er gedenke nach den bevorstehenden Wahlen, ein Datum für den Abzug der amerikanischen Truppen zu fordern. Wenn er dies tut, die Amerikaner sich jedoch weiterhin weigern, ein 
Abzugsdatum festzulegen, dürfte ihre Stellung im Lande noch weit schwieriger werden.

Auch ohne dies wirkt die Zeit zu Ungunsten der Besetzungsmacht, solange es ihr nicht gelingt, Ruhe und Sicherheit im Lande zu schaffen. Gerade dies sucht die Guerrilla, deren Leitung höchstwahrscheinlich in den Händen ehemaliger Geheimdienstleute Saddams liegt, mit allen Mitteln zu verhindern. Die islamistischen Selbstmordkandidaten und Bombenleger sind als ihre Werkzeuge anzusehen. Ihre Zahl nimmt in dem Masse zu, in dem im Lande Wut und Verzweiflung um sich greifen. Irakische Polizisten, Polizeirekruten, Militärs und Rekruten gehören zu den bevorzugten Bombenzielen.

Die Zukunftsaussichten: Die gegenwärtige Entwicklung, die auf eine langsame aber stetige Steigerung der Überfälle und Bombenanschläge hinausläuft, dürfte so weiter gehen, obwohl die Amerikaner sich eine Wende von dem geplanten künftigen Wachstum der irakischen Sicherheitskräfte erhoffen. Man kann eine solche Entwicklung nicht ausschliessen. Sie zu bewerkstelligen, wenn sie je erreicht werden kann, wird jedoch gewiss sehr viel mehr Zeit erfordern, als die Propagandisten der amerikanischen Regierung und Besatzungsbehörden in Aussicht stellen. Und die Wahrscheinlichkeit, dass in jener Zeit auch die Guerilla anwächst, ist gross, weil der Hass auf die Besetzungsbehörden offensichtlich eskaliert. Nicht zu Unrecht machen die Iraker sie für die Zustände verantwortlich, unter denen sie leben müssen.

Dass Washington seinerseits in den nächsten drei Jahren, in denen die Bush-Regierung noch an der Macht sein wird, eine wesentliche Korrektur an seiner Irak Politik vornehmen könnte, ist unwahrscheinlich; denn bis jetzt ist das Bush-Regime nicht bereit, auch nur anzuerkennen, dass es mit seinem Irak-Krieg einen schweren Missgriff getan hat, der unter anderem dazu führt, dass die islamistischen Terroristen weltweit an Zahl und an Zorn zunehmen. Die Bush-Regierung zieht vor, sich selbst und den Amerikanern einzureden, es gehe alles zwar langsamer als erwartet, aber doch letzten Endes ganz gut voran. Die amerikanischen Truppen würden abziehen können, sobald die irakischen an ihre Stelle zu treten vermöchten, und der Irak werde sich am Ende dank der amerikanischen Intervention in eine Demokratie verwandeln.

Die amerikanische Propaganda stützte sich auf die Wahlen von Beginn dieses Jahres, die angeblich „erfolgreich“ durchgeführt wurden, um zu behaupten, die erhoffte Demokratie im Irak stehe vor der Türe, ja sie sei schon verwirklicht. Die Wahlen, an denen sich die meisten Sunniten überhaupt nicht beteiligten, haben jedoch in Wirklichkeit dazu gedient, den Graben zwischen den Sunniten einerseits und den Kurden und Schiiten auf der Gegenseite zu vertiefen. Was den politischen Zielen der "Terroristen“ genau entspricht. Ähnliches hat auch der Dauerstreit über die Verfassung bewirkt. Die angestrebte Demokratie bleibt selbstverständlich solange eine reine Utopie, als die angeblich demokratisch gewählten Behörden nicht für Sicherheit und Ordnung in ihrem Land sorgen können. Die gleiche Voraussetzung gilt auch für eine jede wirtschaftliche Entwicklung. Solange jedoch die Amerikaner ihrer eigenen Propaganda mehr Glauben schenken als der Erkenntnis der himmelschreienden realen Zustände im Irak (für welche sie als Besetzungsmacht rechtlich verantwortlich sind) gilt für sie: Fehler, die nicht erkannt werden, kann man auch nicht korrigieren.

Man muss unter diesen Umständen eine lang hingezogene Krise erwarten, die im schlechtesten aber wohl wahrscheinlicheren Fall schon vor oder erst nach einem Abzug der Amerikaner in einen grausamen Bürgerkrieg im Irak, etwa im Stil Jugoslawiens, ausarten könnte. Im besten, aber weniger wahrscheinlichen Fall einen neuen Starken Mann, mehr oder weniger im Stile Saddams, an die Macht über das ganze Land bringen könnte.

Die Folgen für die umliegenden Länder auf der arabischen und auf der iranischen Seite Iraks sowie im Golf und in der Türkei können nur 
negativ ausfallen. Im schlimmsten Fall könnten die irakischen Unruhen auf sie übergreifen; im besten wäre zu erwarten, dass sie ihre Regime aufrecht zu halten vermögen, jedoch gezwungen sein werden, ihre innere Stabilität durch wenig demokratische Polizeimassnahmen abzusichern.

Ein Israel freilich, das die Landnahmepolitik Ministerpräsident Sharons durch einseitige Massnahmen gegenüber den Palästinensern zur Bildung von palästinensischen Bantustans fortführen will, dürfte von einer Dauerkrise im Irak profitieren. Es könnte sich noch mehr als bereits heute als der einzige zuverlässige Bündnispartner Amerikas profilieren und müsste aus diesem Grunde noch weniger als bereits heute mit einer Intervention der Amerikaner zu Gunsten der theoretisch von ihnen mitvorgesehenen Zwei-Staaten-Lösung im Rahmen der „Road Map“ rechnen, eines Plans, den Sharon zu umgehen versucht.

\section{Fussnoten:}

${ }^{1} \mathrm{C}$ [Dearlove] berichtete über seine soeben beendeten Gespräche in Washington. Es bestehe eine deutliche Veränderung in der Haltung. Ein militärischer Eingriff werde nun als unvermeidlich angesehen. Bush wolle Saddam durch militärische Aktion entfernen, die durch die Zusammenwirkung von Terrorismus und WMDs zu rechtfertigen sei. Aber Informationen und Tatsachen würden entsprechend der politischen Absichten angefertigt.

${ }^{2}$ Israel kann seine strategische Umwelt in Zusammenarbeit mit der Türkei und Jordanien formen, um Syrien einzudämmen oder sogar zurückzudrängen. Diese Bemühungen können darauf ausgehen, Saddam Hussein von der Macht zu entfernen, was in sich selbst schon ein wichtiges strategisches Ziel Israels darstellt. So können die syrischen Pläne frustriert werden. Jordanien hat Syrien kürzlich herausgefordert, indem es die Restauration der Hashemiten im Irak vorschlug. 


\section{Perspektiven des Nahostkonfliktes und die Rolle der Schweiz}

\section{von Laurent Goetschel}

Ich danke ganz herzlich für die Einladung. Es ist in zweifacher Hinsicht eine besondere Ehre: Sie erlauben mir, erstens, die Vortragsreihe 04/05 zu eröffnen. Ich bin in dieser Hinsicht so etwas wie Ihr „Beaujolais nouveau“. Zweitens gaben Sie mir ein Thema, das nicht nur wichtig und von grosser Aktualität ist, sondern das auch heikel und umstritten ist. Dies ist auch der Grund, wieso ich diesen Vortrag ausformuliert habe - etwas, was ich sehr selten mache. Es erlaubt jedoch, auch im Nachhinein genau zu wissen, was man gesagt hat.

Ich werde zuerst über den Nahostkonflikt und seine Perspektiven sprechen und anschliessend über die Rolle der Schweiz. Daraus ergibt sich auch, was ich nicht tun werde: Ich werde nicht über die Geschichte und die Entstehungsgründe des heutigen Konfliktes sprechen. Ich denke, dass wir mit der Aktualität und den sich daraus ergebenden Perspektiven schon mehr als genügend Stoff zum Nachdenken haben.

Noch eine letzte Eingangsbemerkung: Wir haben die Tendenz, den Nahostkonflikt als etwas Besonderes, ja etwas Einzigartiges zu betrachten. Er ist es in einem gewissen Sinne auch, vor allem was dessen Tragweite betrifft. Ich werde jedoch versuchen, auch das "Normale" dieses Konfliktes und die sich daraus ergebenden Konsequenzen herauszuarbeiten. Und ich fange gleich damit an:

Konflikte sind an sich weder etwas Ausserordentliches noch etwas Schlechtes: Überall dort, wo Menschen in Gruppen zusammenleben, kommt es zu Konflikten. In allen Gesellschaften gehört das Aufkommen unterschiedlicher Haltungen zu bestimmten Themen zur Normalität. Solche Meinungsverschiedenheiten gehören sogar zu den fun- 
damentalen Antriebskräften menschlicher Entwicklung. Dialektische Theorien wie der Marxismus haben sie zum Kern ihres Erklärungsansatzes erhoben. Spannungen dieser Art gelten nicht als negativ, ganz im Gegenteil: Sie beinhalten das Potenzial sozialen Wandels und Entwicklung.

Gefährlich aber werden solche sozialen Konflikte erst dann, wenn die Konfliktparteien über eine gewisse Zeitdauer hinweg in unvereinbarer Weise handeln, mindestens eine der beteiligten Parteien die negativen Folgen ihres Handelns auf andere Parteien bewusst in Kauf nimmt, und wenn eine der beteiligten Parteien Schaden aus den Handlungen zieht. Das Vorhandensein eines solchen Konfliktes setzt somit absichtliches und mit Schadenszufügung verbundenes Handeln voraus.

Diese akademisch klingende Definition erwähne ich nicht, weil ich glaube, beweisen zu müssen, dass wir es im Nahen Osten wirklich mit einem Konflikt zu tun haben. Soweit sind wir uns wohl alle einig. Ich erwähne sie deswegen, weil mir daran liegt, zu unterstreichen, dass Interessengegensätze, sogar schwere, nicht zwingend zu Gewaltanwendung führen müssen. Es gibt offensichtliche Interessengegensätze im Nahen Osten: Sie haben zu tun mit territorialen Ansprüchen, mit religiöser und kultureller Identität, mit dem Zugang zu erneuerbaren und nicht-erneuerbaren natürlichen Ressourcen (Wasser und Öl) und auch mit ökonomischen Interessen. Trotz dieser Vielzahl an Gegensätzen gibt es aber keinen zwingenden Grund, dass der Konflikt gewaltsam ausgetragen werden muss, wie dies zurzeit der Fall ist. Das Vorhandensein eines solchen Gewaltkonfliktes bedingt absichtliches und mit Schadenszufügung verbundenes Handeln. Es spielt dabei keine Rolle, wer wen wofür "bestraft" oder wer auf welche Handlung wie „reagiert": Bei einem Konflikt, der seit mehr als 50 Jahren dauert, ist die Frage, wer „angefangen“ hat, irrelevant. Es gibt keine „Unschuldigen“. Damit Sie mich richtig verstehen: Ich meine damit die Konfliktparteien als Kollektive. Ich meine nicht die zahlreichen un- schuldigen Opfer, Kinder, Frauen und Männer, auf beiden Seiten.

Ein konstruktiver Umgang mit der heutigen Konfliktsituation setzt voraus, erstens, dass die Konfliktparteien miteinander reden und sich auf die wichtigsten Konfliktinhalte einigen. Sie sollten dann, zweitens, einen Prozess definieren, der es innen erlaubt, ohne Anwendung von Gewalt über diese Konfliktinhalte zu diskutieren. Als Ergebnis davon sollten, drittens, Lösungen für die wichtigsten Konfliktdimensionen gefunden werden. Es ist dies der einzige Weg, der nachhaltig zu einer Reduktion der Gewalt und einer freien Entfaltung der Menschen in der Konfliktregion führen wird.

Zum ersten Punkt: Die Konfliktparteien müssen miteinander reden. Es ist dies ein banaler Satz. Aber er beinhaltet zwei essentielle Bedingungen: Es geht um die Konfliktparteien. Und sie müssen miteinander reden. Man spricht, so harsch dies klingen mag, mit dem Feind. Diesen kann man nicht auswählen. Er konstituiert sich selber. Man trägt durch das eigene Verhalten das eine oder andere dazu bei, aber das ist eine andere Geschichte. Auf Seiten Israels ist dieser Gesprächspartner der Regierungschef, auf Seiten der Palästinenser der Präsident der Autonomiebehörde. Wenn man ein Gespräch auf dieser obersten offiziellen Ebene sucht, gibt es diesbezüglich keinen Handlungsspielraum. Bedingungen wie „wir wollen reden, aber nicht mit ihm...", machen keinen Sinn. Sie bedeuten im Grunde genommen nichts anderes als: "wir wollen vorderhand nicht miteinander reden“. Wenn dem so ist, soll man dies auch so kommunizieren. Das Ergebnis ist ohnehin dasselbe, nämlich dass keine Gespräche stattfinden.

Wenn die Gespräche nicht auf dieser offiziellen Eben stattfinden (Track 1), gibt es mehr Handlungsspielraum: Man kann mit Personen reden, die der politischen Führung „nahe stehen“ oder sonst einflussreich sind. Je weiter weg man sich vom innersten Entscheidzirkel begibt, umso "gesprächsbereiter" werden die „Unterhändler" sein (Track $1,5-3)$, umso innovativer werden die potenziellen Themen und die 
Lösungsvorschläge. Leider nimmt damit auch der direkte politische Einfluss der Gesprächspartner ab.

Trotzdem oder deswegen können solche Gespräche in bestimmten Situationen sinnvoll sein, weil damit auch Themen aufgegriffen werden, die sonst verdrängt werden (Tabu-Themen). Auch ist dies manchmal die einzige Möglichkeit, überhaupt Gespräche zu führen. Schliesslich können damit neue Perspektiven eröffnet werden. Sie merken es, dies ist eine Anspielung auf die Genfer Initiative. Ich werde später darauf zurückkommen.

Eigentlich war dies nur ein Vorspann zu meinem ersten Punkt: Dieser handelt von den Konfliktinhalten (über die man reden muss, um sich darauf zu einigen). Im Fall des Nahen Ostens ist der wichtigste Punkt zweifellos derjenige des Landes: Der Nahostkonflikt ist in erster Linie ein territorialer Konflikt. Die meisten anderen wichtigen Probleme sind Folgen dieses Land-Disputs: Es geht um Flüchtlinge (Rückkehrrecht), um Land-Eigentumsrechte (Siedlungen etc.), um Grenzen.

Es führt kein Weg an der Einsicht vorbei, dass grundsätzlich weite Teile desselben Territoriums von mindestens zwei verschiedenen Volksgruppen beansprucht werden (Beduinen, Drusen,...). Und wenn von palästinensischer Seite der Rückstoss der Israelis ins Mittelmeer und von israelischer Seite die Auswanderungsoption für die Palästinenser nach Jordanien erwähnt werden, dann untermauert dies nur die Zentralität dieser territorialen Frage. Zugleich ist dies ein Beleg für die nach wie vor auf beiden Seiten bestehende Bereitschaft, die Realität zu verdrängen und illusionären Hoffnungen nachzuhängen! In anderen Zusammenhängen ist diesbezüglich von „Problemverdrängung" die Rede: je schlimmer die Situation, umso grösser die Verdrängung, ein Verhalten, dass pathologische Züge annehmen kann...

Zum zweiten Punkt: Es muss ein Prozess definiert werden, der es erlaubt, ohne Anwendung von Gewalt über Konfliktinhalte und deren
Lösungen zu diskutieren. Dieser muss beidseitig akzeptiert sein. Er muss glaubwürdig sein. Er kann mit oder ohne Mitwirkung von Drittparteien zustande kommen. Er kann sich auch mit oder ohne Mitwirkung Dritter abwickeln.

In einem Kontext wie demjenigen des Nahostkonfliktes ist es vor allem wichtig, dass ein solcher Prozess Perspektiven eröffnet! Wenn ich mir überlege, was ich am Nahostkonflikt als wirklich erschütternd betrachte, dann ist es seine Perspektivlosigkeit. Ich sehe zurzeit keine sich abzeichnenden Perspektiven für einen nachhaltigen, konstruktiven Umgang mit der Situation. Starke Kräfte auf beiden Seiten profitieren vom Stillstand des Friedensprozesses und vom Teufelskreis von Gewalt und Gegengewalt. Es gab Ansätze: der Oslo-Prozess, die Road-Map und andere mehr. Aber Sie wissen genauso gut wie ich, dass diesen Vorhaben zurzeit nicht sonderlich viel Bedeutung beigemessen wird. Der in Aussicht gestellte unilaterale Abzug israelischer Truppen aus dem Gaza-Streifen bietet keine solche Perspektive.

Und diese Perspektivlosigkeit, meine Damen und Herren, ist für die palästinensische Bevölkerung um ein Vielfaches eklatanter, als dies für die israelische Bevölkerung der Fall ist. Jugendliche Palästinenser (rund 2/3 der Bevölkerung) können sich nicht in die Unterhaltungswelt von Tel Aviv oder Haïfa flüchten. Sie haben keine beruflichen Perspektiven, sind in ihrer Bewegungsfreiheit extrem eingeschränkt. Sie haben im Grunde genommen keine Zukunft. Diese wird innen verbaut, sowohl im übertragenen Sinne als auch sprichwörtlich.

Und damit komme ich zum Thema des Terrorismus: Terrorismus ist eine Form der Konfliktaustragung. Es gibt keinen Terrorismus ohne Konflikt. Bei dieser Form der Konfliktaustragung wird punktuell und unvorhersehbar, aber systematisch Gewalt gegen zivile Ziele ausgeübt. Ziel ist es, den Staat zu provozieren und zu einer Überreaktion zu veranlassen. Wenn der Staat sich dann dazu hergibt, mit Gegengewalt zu antworten und sich dabei nicht rechtstaatlicher Mittel bedient, haben die Terroristen ihr Ziel erreicht: Sie haben bewiesen, dass der 
Machtapparat die Anliegen der von innen „repräsentierten“ diskriminierten Bevölkerungsgruppen nicht ernst nimmt. Er verdient folglich nichts anderes, als mit Waffengewalt bekämpft zu werden. So wird mit Gewalt und Gegengewalt die Eskalationsspirale in Gang gehalten. Gleichzeitig führt die Unverhältnismässigkeit der Reaktion des staatlichen Machtapparates den Terroristen neue Mitglieder zu. Diese Zeilen stammen aus dem Vortrag eines meier Kollegen bei swisspeace, Heinz Krummenacher. Er hat diesen Vortrag kürzlich in Hamburg zum Angriff auf die nordossetische Schule in Beslan gehalten. Terrorismus hat es seit jeher gegeben. Politisch, religiös oder sozial-revolutionär motivierte Gruppen suchten Zuflucht zu dieser Kampfform, weil sie meinten, keine andere Wahl zu haben. Ich überlasse es Ihnen, die Parallelen zum Nahostkonflikt zu ziehen.

Meinerseits belasse ich es bei der Forderung, baldmöglichst glaubwürdige Perspektiven für einen Friedensprozess aufzuzeigen. Ich weiss, dass dies nicht einfach ist. Doch das ist noch lange kein Grund, dies nicht zu fordern, vor allem dann, wenn keine sinnvolle Alternative in Sicht ist.

Ich komme zum dritten Punkt: den Lösungsansätzen. Der Teilungsplan der zuständigen UNO-Kommission aus dem Jahre 1947 war einer der ersten Lösungsansätze für den Territorialkonflikt im Nahen Osten. Er ging, wie der Name es sagt, von einer Zweistaaten-Lösung aus. Diese stützte sich auf die damals bestehenden Schwerpunkte der Siedlungsgebiete beider Bevölkerungsteile. Jerusalem sollte von einer internationalen Treuhandschaft verwaltet werden. Die damals unterlegene Kommissions-Minderheit sah die Gründung eines palästinensischen Bundesstaates vor, der je aus einem arabischen und einem jüdischen Teilstaat bestehen sollte, mit Jerusalem als Hauptstadt.

Seither hat sich in der Region vieles verändert:
- Mit dem Sechstage-Krieg von 1967 übernahm Israel die Kontrolle über Gebiete, die mittlerweile von mehreren Millionen Palästinensern bewohnt werden.

- Mit Ägypten und Jordanien wurde der Kriegszustand überwunden.

- Seit Beginn der ersten Intifada führt Israel einen Krieg nicht mehr gegen arabische Nachbarstaaten, sondern innerhalb des von ihm besetzten und kontrollierten Territoriums.

An Lösungsansätzen für den Konflikt hat es seit 1947 nicht gemangelt. Mit dem Oslo-Prozess Anfang der 90er Jahre gab Israel erstmals offiziell seine Opposition gegen die Errichtung eines palästinensischen Staates auf. Diese sogenannte „Zwei-Staaten-Lösung“ bildete auch die Grundlage für die meisten seither propagierten Lösungen (bspw. International Crisis Group, ICG).

Wenn ich jetzt die Möglichkeit hätte, alle meine Wünsche anzubringen, würde ich für eine Einstaaten- oder sehr durchlässige ZweiStaaten-Lösung optieren:

- Eine solche würde es beiden Bevölkerungsgruppen erlauben, dort zu leben, wo sie wollen, ob in der Westbank oder im Kernland Israels

- Alle hätten dieselben demokratischen Rechte

- Israel würde als Vorbild einer integrierten, pluri-religiösen, politischen Gemeinschaft in die Geschichte des Nahen Ostens eingehen...

Wir wissen, leider, dass dies keine realistische Perspektive ist: Künstler, wie Daniel Barenboïm, mögen dies offen und mit guten Absichten propagieren. Er preist, wie vor wenigen Wochen in Genf, das Zusammenwirken seines gemischt israelisch-palästinensischen Orchesters als Beispiel für die Machbarkeit einer Einstaaten-Lösung an. Doch ein 
Staat ist (leider) weniger harmonischen Prinzipien unterworfen als ein Orchester.

Gleiche Rechte für die Palästinenser in einem einheitlichen Staat würde bedeuten, dass diese aufgrund ihrer viel höheren Geburtenrate in absehbarer Zeit die Bevölkerungsmehrheit darstellen würden. Sie könnten damit auf ganz demokratische Art und Weise auch die Regierungsmehrheit bilden. Dies wäre in einem anderen politischen Umfeld an sich kein unüberwindbares Problem: Es gibt genügend institutionelle politische und rechtliche Instrumente, die das Zusammenleben unterschiedlicher Bevölkerungsgruppen in ein und demselben Staatswesen ermöglichen. Gerade in der Schweiz verfügen wir diesbezüglich über einen beträchtlichen Erfahrungsschatz. Aber solche politische Modelle können nicht einfach "exportiert" werden. Zwischen den Juden und den Palästinensern in Israel und in Palästina fehlt eine elementare Vertrauensbasis, um eine solche EinstaatenLösung ins Auge fassen zu können.

Es ist nicht auszuschliessen, dass diese Vertrauensbasis in einer wohl eher fernen als nahen Zukunft entstehen könnte. Ich fände dies als Ziel durchaus erstrebenswert. Man stelle sich vor: Israel als Vorbild für das Zusammenleben von Juden, Christen und Moslems. Dies wäre ein konkreter Beitrag zum Frieden nicht nur in der Region, sondern in der Welt. Natürlich würden die Heiligen Stätten in Jerusalem paritätisch von Vertretern der drei Religionen verwaltet. Utopien sind erlaubt. Sie sind manchmal sogar notwendig.

Doch der nächste Schritt wird mit an Sicherheit grenzender Wahrscheinlichkeit eine Zweistaaten-Lösung sein. Alles andere wäre bei weitem verfrüht. Wir können auch einen (grossen) Schritt zurückmachen und uns überlegen, wie mit den unmittelbar anstehenden Problemen umgegangen werden sollte, um die Utopie nicht aus den Augen zu verlieren, oder in anderen Worten: Wie kann das Vertrauen zwischen beiden Bevölkerungsgruppen langsam aufgebaut werden?

Und hier kommt das zweitgrösste Problem des Nahostkonfliktes zur Sprache: Der Einfluss der Religionen. Ich hatte vorher ja bereits gesagt, dass es sich beim Nahostkonflikt primär um einen territorialen Konflikt handelt. Das stimmt auch. Der Umgang mit diesem territorialen Disput wird jedoch ganz entscheidend dadurch erschwert, dass auf beiden Seiten religiöser Eifer einen konstruktiven Lösungsansatz erschwert.

Bitte verstehen Sie mich nicht falsch: Es geht nicht um die Religionen an sich. Sie sind Bestandteil des Konfliktes und müssen daher auch Bestandteil jeglicher Lösung sein. Meine zuvor kurz skizzierte Utopie eines gemeinsamen Staatswesens besteht ja nicht zuletzt im Kern in der politischen Ko-Existenz der verschiedenen Glaubensgemeinschaften.

Aber auf beiden Seiten vorhandene Extremlösungen werden allzu oft aus religiösen Verheissungen und Vorstellungen gespiesen. Wenn ein Jude das Gefühl hat, er tue etwas Gutes, wenn er in Israel wohne, ist das eine und auch seine Sache. Wenn er jedoch überzeugt ist, er tue etwas noch viel Besseres, wenn er sich in der Westbank, oder eben Judäa und Samaria niederlasse, ist dies etwas ganz anderes.

Siedler gibt es auch in nordöstlichen indischen Gliedstaaten und im Osten Äthiopiens (dort wo Lucy herkommt....). Es gab sie in den Geschichten Südafrikas und der USA. Man spricht diesbezüglich auch von „Besiedlung“. Damit wird insinuiert, dass zuvor keine Menschen in den entsprechenden Gegenden anwesend waren, was auf keines der erwähnten Beispiele zutrifft. Zumeist ist in solchen Gebieten das Staatswesen nicht so gefestigt, wie in den Kernlanden. Das Rechtsgefühl ist etwas "schwammiger", und damit die „Rechts-Freiheit" (wohlverstanden diejenige der Siedler) etwas grösser. Man sorgt selber für „Ruhe und Ordnung“ und tut dies oft mit der Überzeugung, damit eine wie auch immer definierte Mission zu erfüllen. Manchmal sind es jedoch auch ganz banale finanzielle Anreize. Siedler haben noch nirgends auf der Welt Friedensprozesse vorangetrieben. Allenfalls haben sie ansässige Bevölkerungen vertrieben oder ausgerottet 
und damit auf ihre Art und Weise für einen Frieden gesorgt, der seinen Namen nicht verdient.

Sich auserwählt zu fühlen, heisst nicht, ausgenommen zu sein, besondere Rechte zu haben, oder ähnliches. Wenn Auserwähltheit irgendeinen Sinn haben soll, dann ist es derjenige, besonderen Pflichten unterworfen zu sein. Diese sollten zu einem exemplarischen Denken und hoffentlich auch Verhalten führen. Davon ist, im Nahen Osten zurzeit herzlich wenig zu spüren. Man bekommt eher den Eindruck, dass je auserwählter sich die Personen fühlen, umso weniger exemplarisch sie sich verhalten.

Damit komme ich zur Rolle der Schweiz: Was kann die Schweiz in Bezug auf die aufgezeigten, nicht sonderlich erbaulichen Perspektiven des Nahostkonfliktes unternehmen? Was sollte sie machen?

Die Schweiz hat drei Besonderheiten aufzuweisen, die mit Blick auf den Nahostkonflikt von Interesse sein könnten:

- $\quad$ Sie geniesst den Ruf eines kleinen und neutralen Staates, der keine eigenen machtpolitischen Interessen verfolgt.

- Sie ist Depositarstaat der Genfer Konventionen und hat damit in Bezug auf die Einhaltung des Humanitären Völkerrechtes eine besondere, wenn auch nicht abschliessend definierte Rolle zu spielen.

- Sie ist, schliesslich, traditionell Wohnort und auch Heimatland zahlreicher Diasporas.

Diese Besonderheiten müssen in Bezug gestellt werden zu den Anforderungen, die ich vorher erwähnt habe: 1) Parteien müssen miteinander reden, 2) Perspektiven müssen aufgezeigt werden, 3) Lösungen müssen erarbeitet und akzeptiert werden.

Wie steht es um die Vermittlung der neutralen und kleinen Schweiz? Hierzu will ich zunächst mal klarstellen, dass jegliche bewusste Handlung einer Drittpartei, welche die Beeinflussung eines Konfliktver- laufs zum Ziel hat, eine Intervention ist. Jede Intervention verändert zwangsläufig auch das Machtverhältnis zwischen den Parteien. Es gibt diesbezüglich keine neutralen Interventionen. Oder anders gesagt: Neutralität spielt für die Intervention in einen Konflikt keine Rolle. Neutrale Staaten haben in den vergangenen Jahrzehnten auch nicht häufiger oder erfolgreicher vermittelt als andere Länder.

Hinzu kommt, dass sich die Schweiz vor allem als Anbieterin sogenannter "Guten Dienste" einen Ruf erworben hat: Solche Dienste zielen darauf ab, Verhandlungen (logistisch) zu organisieren oder, im Falle einer staatlichen Drittpartei, ihr Territorium als Verhandlungsort zur Verfügung zu stellen. Traditionellerweise beinhalteten sie Aktivitäten, welche die Konfliktparteien einander näher bringen sollen, ohne damit politischen Einfluss auszuüben. Diese Art von Leistungen ist im Nahostkonflikt zurzeit wenig gefragt. Es ist nicht so, dass die Parteien nicht wüssten, wo man sich treffen könnte, sondern dass die Gespräche inhaltlich und prozedural blockiert sind. Mit Sicherheit würde die Schweiz jedoch ihr Territorium für allfällige Gespräche zur Verfügung stellen wollen.

Sofern die Vermittlung nicht nur passiv Gelegenheiten schafft, sondern auch aktiv den Informationsaustausch zwischen den Konfliktparteien fördert, ist von „Facilitation" die Rede. In einer weiteren Stufe kann eine solche Funktion zur Übernahme der Rolle eines Moderators führen. Hierfür dürfte in erster Linie eine grössere Macht als die Schweiz in Frage kommen, primär die USA, die Europäische Union oder einer ihrer grösseren Mitgliedstaaten. Aber zunächst mal müssten die Parteien an den Verhandlungstisch zurückkehren.

Wie steht es um das Aufzeigen von Perspektiven? Hier sehe ich einen sehr wichtigen Beitrag, den die Schweiz mit ihrer Unterstützung der "Genfer Initiative" geleistet hat. Deren Kern besteht aus einem Friedensplan für den Nahostkonflikt, dem Genfer Abkommen, das von nicht offiziellen Vertreterinnen und Vertretern der Israelis und der Palästinenser ausgehandelt worden ist. Das grösste Verdienst der In- 
itiative bestand aus meiner Sicht darin, dass sie, erstens, aufzeigte, dass es auf beiden Seiten Personen gibt, auch nicht ganz unwichtige, die zu Verhandlungen bereit sind. Zweitens zeigte sie die Machbarkeit, „unvollendete“ Verhandlungen zu Ende zu führen und damit auch heikle Themen auszudiskutieren. Dass gewisse Einzelheiten ungeregelt blieben, ist in diesem Zusammenhang sekundär.

In der Kritik am Genfer Abkommen wurde auch klar, welches die wahren Knacknüsse sind. Ein gutes Beispiel ist das Rückkehrrecht für geflohene Palästinenser: Es ist völlig unrealistisch, zu erwarten, dass alle geflohenen Palästinenser nach Israel zurückkehren könnten. Die Frage ist jedoch weniger, ob dieses Recht umgesetzt wird oder nicht, sondern dass es anerkannt wird. Danach gibt es verschiedene denkbare Formen der Entschädigung, ganz abgesehen davon, dass kaum alle geflohenen Palästinenser und Palästinenserinnen nach Israel zurückkehren möchten. Sollte es künftig zu vertieften Verhandlungen dieses Themas kommen, fände ich es für die Schweiz eine Herausforderung, darin eine bedeutende Rolle wahrzunehmen.

Die grosse Aufmerksamkeit, die dem Genfer Abkommen im internationalen Umfeld, aber auch in der Region zuteil wurde, zeigt, wie "ausgetrocknet" der Markt an Friedens-Perspektiven ist. Indem die Schweiz half, das Abkommen auf eine internationale Ebene zu hieven, hat sie Bedeutendes zur Visibilität des Vorschlags beigetragen.

Ich komme zum nächsten Punkt, demjenigen des Humanitären Völkerrechts. Im Grunde genommen ist dieses Recht schnell erklärt: Es gewährleistet Mindestnormen zum Schutz des Individuums im Umfeld von Kriegen. Darunter fällt auch der Umgang mit Gebieten, die in Kriegen besetzt und weder annektiert noch zurückgegeben wurden. Während bestimmten dieser Normen im Krieg zentrale humanitäre Funktionen zukommen, setzen andere Anforderungen an den Umgang mit besetzten Gebieten, die gewährleisten sollen, dass die Bevölkerungen dieser Gebiete nicht unter der Willkür der Besetzungsmacht leiden. Das humanitäre Völkerrecht gilt als Bestandteil des zwingenden Völkerrechts mit Bindungen erga omnes. Das heisst, dass die Einhaltung der entsprechenden Normen keine Gegenseitigkeit voraussetzt: Man kann sich nicht hinter Fehlleistungen der Gegenseite verstecken, um eigene Unzulänglichkeiten zu rechtfertigen.

Ich will mich hier nicht in rechtliche Einzelheiten verlieren, sondern nur folgendes betonen: Die Grundsätze des humanitären Völkerrechts bezwecken, die Einhaltung absoluter Minimal-Standards sicherzustellen. Wird - mit welchem Zeithorizont auch immer - ein Zusammenleben der Israelis und Palästinenser angestrebt, so sollten schon heutzutage möglichst wenige Handlungen erfolgen, die eine Verletzung dieser Rechtsnomen beinhalten. Der Verlauf der Mauer (oder des Zaunes) bzw. der "Sperranlage“ im Umfeld der ehemaligen "Grünen Linie“ bietet hierfür kein gutes Beispiel. Das Verüben terroristischer Akte durch Palästinenser ebenso wenig.

Vor diesem Hintergrund sehe ich den Einsatz der Schweiz zur Einhaltung des humanitären Völkerrechts als sehr bedeutend an. Mag er manchmal technokratisch oder pedantisch erscheinen, er dreht sich um absolute Grundsätze im gegenseitigen Umgang der Konfliktparteien. Er ist damit zumindest ein indirekter Beitrag zum Frieden im Nahen Osten.

Ich komme zum letzten Punkt: Es geht um die Bedeutung der Diasporas, die in der Schweiz ansässig sind. Diese gehören auch zur Schweiz, unabhängig ihrer Nationalität. Es gibt zahlreiche einflussreiche Personen, und zwar sowohl auf palästinensischer als auch auf israelischer und jüdischer Seite, die durchaus über Kanäle zu massgebenden Akteuren auf beiden Seiten des Nahostkonfliktes verfügen. Diese können sogar besser als diejenigen der offiziellen Schweiz sein.

Es hindert uns somit nichts daran, innerhalb der Schweiz den Nahost-Dialog einzuüben. Dies ist allerdings leichter gesagt als getan. Denn um Dialogfähigkeit zwischen Gruppen zu erreichen, braucht es zunächst Dialogfähigkeit innerhalb der Gruppen. Sie wissen, was ich 
meine: Es geht darum, innerhalb von Palästinensergruppen und innerhalb jüdischer Kreise zu einem minimalen Konsens zu gelangen. Dies ist alles andere als einfach, und zwar auf beiden Seiten.

Ich bin jedoch der Ansicht, dass hier ein nicht zu unterschätzendes Potenzial liegt: Werden Dialogprozesse innerhalb der Schweiz in Gang gebracht, können mit der Zeit auch Vertreter und Vertreterinnen aus der Region beigezogen werden. Solche Gespräche, von denen es übrigens schon einige gegeben hat, können dazu dienen, neue Ideen, aber auch Konsensvorschläge für konkrete Problembereiche, wie beispielsweise denjenigen der Flüchtlingsfrage, auszuarbeiten. Es könnte auch ein gemeinsames Verständnis der Geschichte des Nahen Ostens gefördert werden, etwas, das für die Suche nach Lösungen von essentieller Bedeutung ist.

Solche Gespräche zwischen Diasporas in der Schweiz könnten somit helfen, sowohl Perspektiven aufzuzeigen als auch Testgründe für die Erarbeitung konkreter Lösungsvorschläge in einzelnen Teilbereichen zu sein.

Zugleich verfolgt die Schweiz auf staatlicher und nicht-staatlicher Ebene zahlreiche Projekte im Bereich der Entwicklungszusammenarbeit, der humanitären Hilfe und der zivilen Friedensförderung in der Region. Diese Projekte haben alle ihren eigenständigen Wert, vor allem wenn sie dazu dienen, marginalisierte Gruppen zu fördern, unter anderem Frauen, oder das gegenseitige Verständnis zwischen den Parteien aufzubauen. Diesbezüglich sind Jugend- und Austauschprojekte von besonderer Bedeutung.

Meine Damen und Herren, liebe Freunde, ich habe versucht, durch meinen ganzen Vortrag hinweg die Bedeutung folgender Punkte zu unterstreichen: Reden, Perspektiven, Lösungsvorschläge. Der Nahostkonflikt mag seiner Tragweite wegen einzigartig sein, abgesehen davon aber ist er es nicht. Man kann einen Konflikt nicht alleine lösen und auch nicht unilateral für beendet erklären. Man braucht ab und zu Visionen, um die Orientierung nicht zu verlieren. Auch hier in der
Schweiz haben wir die Möglichkeit, unter uns und über Beziehungsnetze, über die wir alle verfügen, an Perspektiven und Lösungsoptionen für den Nahostkonflikt zu arbeiten. Ich hoffe, mit meinem Vortrag einen weiteren Impuls in diese Richtung gegeben zu haben und danke Ihnen fürs Zuhören. 


\section{BASLER SCHRIFTEN ZUR EUROPÄISCHEN INTEGRATION}

O Wir bestellen die Schriftenreihe im Jahresabonnement zu CHF 120.-. Das Abonnement verlängert sich automatisch um ein Jahr, wenn es nicht drei Monate vor Ablauf schriftlich gekündigt wird.

O Wir bestellen folgende Nummern zum Preis von CHF 20.- (Doppelnr 30.-)

O Wir sind an einem Publikationsaustausch interessiert.

O Wir sind an Weiterbildungs-Unterlagen (Nachdiplomkurs) interessiert.

* vergriffen

O Nr. 1 Subsidiarität - Schlagwort oder Kurskorrektur (mit Beiträgen von Flavio Cotti, Jean-Paul Heider, Jakob Kellenberger und Erwin Teufel) (Doppelnummer).*

ONr. 2 Ein schweizerisches Börsengesetz im europäischen Kontext (Tagungsband/Doppelnummer).*

O Nr. 3 Martin Holland, The European Union's Common Foreign and Security Policy: The Joint Action Toward South Africa.*

O Nr. 4 Brigid Gavin, The Implications of the Uruguay Round for the Common Agricultural Policy.

O Nr. 6 Urs Saxer, Die Zukunft des Nationalstaates.

ONr. 7 Frank Emmert, Lange Stange im Nebel oder neue Strategie? Die aktuelle Rechtsprechung des EuGH zur Warenverkehrsfreiheit.

ONr. 8 Stephan Kux, Subsidiarity and the Environment: Implementing International Agreements.

ONr. 9 Arbeitslosigkeit (mit Beiträgen von Christopher Boyd, Wolfgang Franz und Jean-Luc Nordmann).

O Nr.10 Peter Schmidt, Die aussenpolitische Rolle Deutschlands im neuen Europa.

O Nr.11 Hans Baumann, Möglichkeiten und Grenzen der Sozialen Dimension nach Maastricht: Das Beispiel der Bauwirtschaft. *

O Nr.12 Georg Kreis, Das schweizerische Staatsvertragsreferendum: Wechselspiel zwischen indirekter und direkter Demokratie.

ONr.13 Markus Lusser, Die europäische Währungsintegration und die Schweiz.
O Nr.14 Claus Leggewie, Ist kulturelle Koexistenz lernbar?

O Nr.15 Rolf Lüpke, Die Durchsetzung strengerer einzelstaatlicher Umweltschutznormen im Gemeinschaftsrecht (Doppelnummer).O Nr.16 Stephan Kux, Ursachen und Lösungsansätze des Balkankonflikts: Folgerungen für das Abkommen von Dayton

O Nr.17 Jan Dietze/Dominik Schnichels, Die aktuelle Rechtssprechung des Europäischen Gerichtshofes zum Europäischen Gerichtsstands- und Vollstreckungsübereinkommen (EuGVÜ).

O Nr.18 Basler Thesen für die künftige Verfassung Europas (2. Aufl.).

O Nr.19 Christian Garbe, Subsidiarity and European Environmental Policy: An Economic Perspective.

O Nr.20 Claudia Weiss, Die Schweiz und die Europäische Menschenrechtskonvention: Die Haltung des Parlaments 1969-1995.

ONr.21 Gunther Teubner, Globale Bukowina: Zur Emergenz eines transnationalen Rechtspluralismus.

ONr.22 Jürgen Mittelstrass, Stichwort Interdisziplinarität (mit einem anschliessenden Werkstattgespräch).

O Nr.23 William James Adams, The Political Economy of French Agriculture.

O Nr.24 Aktuelle Fragen der Wirtschafts- und Währungsunion (mit Beiträgen von Gunter Baer, Peter Bofinger, Renate Ohr und Georg Rich) (Tagungsband/Doppelnummer).

O Nr.25 Franz Blankart, Handel und Menschenrechte.

O Nr.26 Manfred Dammeyer/Christoph Koellreuter, Die Globalisierung der Wirtschaft als Herausforderung an die Regionen Europas.

ONr.27 Beat Sitter-Liver, Von Macht und Verantwortung in der Wissenschaft.

O Nr.28 Hartwig Isernhagen, Interdisziplinarität und die gesellschaftliche Rolle der Geistes- und Kulturwissenschaften.

O Nr.29 Muriel Peneveyre, La réglementation prudentielle des banques dans I’Union Européenne.

O Nr.30 Giuseppe Callovi/Roland Schärer/Georg Kreis, Citoyenneté et naturalisations en Europe.

O Nr.31 Peter Häberle, Gemeineuropäisches Verfassungsrecht. 
O Nr.32 Jacques Pelkmans, Europe's Rediscovery of Asia. Political, economic and institutional aspects.

O Nr.33 Maya Krell, Euro-mediterrane Partnerschaft. Die Chancen des Stabilitätstransfers.

O Nr.34 Valéry Giscard d'Estaing, L'Union Européenne: Elargissement ou approfondissement?

O Nr.35 Martin Holland, Do Acronyms Matter? The Future of ACP-EU Relations and the Developing World

O Nr.36 Andreas Guski, Westeuropa - Osteuropa: Aspekte einer problematischen Nachbarschaft.

O Nr.37 Matthias Amgwerd, Autonomer Nachvollzug von EU-Recht durch die Schweiz - unter spezieller Berücksichtigung des Kartellrechts (Doppelnr.)

O Nr.38 Manfred Rist, Infotainment oder Sachinformation? Die Europäische Union als journalistische Herausforderung (Doppelnummer).

O Nr.39 Lothar Kettenacker/Hansgerd Schulte/Christoph Weckerle, Kulturpräsenz im Ausland. Deutschland, Frankreich, Schweiz.

O Nr.40 Georg Kreis/Andreas Auer /Christoph Koellreuter, Die Zukunft der Schweiz in Europa? Schweizerische Informationstagung vom 15. April 1999 veranstaltet durch das EUROPA FORUM LUZERN.

ONr.41 Charles Liebherr, Regulierung der audiovisuellen Industrie in der Europäischen Union.

ONr.42/3 Urs Saxer, Kosovo und das Völkerrecht. Ein Konfliktmanagement im Spannungsfeld von Menschenrechten, kollektiver Sicherheit und Unilateralismus.

O Nr.44/5 Gabriela Arnold, Sollen Parallelimporte von Arzneimitteln zugelassen werden? Eine Analyse der Situation in der Europäischen Union mit Folgerungen für die Schweiz.

ONr.46 Markus Freitag, Die politischen Rahmenbedingungen des Euro: Glaubwürdige Weichenstellungen oder Gefahr möglicher Entgleisungen?

O Nr.47/8 Andrew Watt, „What has Become of Employment Policy?“ - Explaning the Ineffectiveness of Employment Policy in the European Union.

O Nr.49 Christian Busse, Österreich contra Europäische Union - Eine rechtliche Beurteilung der Reaktionen der EU und ihrer Mitgliedstaaten auf die Regierungsbeteiligung der FPÖ in Österreich.

O Nr.50 Thomas Gisselbrecht, Besteuerung von Zinserträgen in der Europäischen Union - Abschied vom Schweizerischen Bankgeheimnis?

O Nr.51 Uta Hühn, Die Waffen der Frauen: Der Fall Kreil - erneuter Anlass zum Konflikt zwischen europäischer und deutscher Gerichtsbarkeit? EuGH, Urteil vom 11.1.2000 in der Rs. C-283/98, Tanja Kreil/ $B R D$.

O Nr.52/3 Thomas Oberer, Die innenpolitische Genehmigung der bilateralen Verträge Schweiz - EU: Wende oder Ausnahme bei aussenpolitischen Vorlagen?

O Nr.54 Georg Kreis, Gibraltar: ein Teil Europas - Imperiale oder nationale Besitzansprüche und evolutive Streiterledigung.

O Nr.55 Beat Kappeler, Europäische Staatlichkeit und das stumme Unbehagen in der Schweiz. Mit Kommentaren von Laurent Goetschel und Rolf Weder.

ONr.56 Gürsel Demirok, How could the relations between Turkey and the European Union be improved?

O Nr.57 Magdalena Bernath, Die Europäische Politische Gemeinschaft. Ein erster Versuch für eine gemeinsame europäische Aussenpolitik

O Nr.58 Lars Knuchel, Mittlerin und manches mehr. Die Rolle der Europäischen Kommission bei den Beitrittsverhandlungen zur Osterweiterung der Europäischen Union. Eine Zwischenbilanz.

O Nr.59 Perspektiven auf Europa. Mit Beiträgen von Hartwig Isernhagen und Annemarie Pieper.

O Nr.60 Die Bedeutung einer lingua franca für Europa. Mit Beiträgen von Georges Lüdi und Anne Theme.

O Nr.61 Felix Dinger, What shall we do with the drunken sailor? EC Competition Law and Maritime Transport.

O Nr.62 Georg F. Krayer, Spielraum für Bankegoismen in der EU-Bankenordnung.

O Nr.63 Philippe Nell, China's Accession to the WTO: Challenges ahead.

ONr.64 Andreas R. Ziegler, Wechselwirkung zwischen Bilateralismus und Multilateralismus Das Beispiel der Freihandelsabkommen der EFTA- 
Staaten.

O Nr.65 Markus M. Haefliger, Die europäische Konfliktintervention im burundischen Bürgerkrieg, („Arusha-Prozess“) von 1996 bis 2002.

O Nr.66 Georg Kreis (Hrsg), Orlando Budelacci (Redaktion): Der Beitrag der Wissenschaften zur künftigen Verfassung der EU. Interdisziplinäres Verfassungssymposium anlässlich des 10 Jahre Jubiläums des Europainstituts der Universität Basel.

ONr.67 Francis Cheneval, Die Europäische Union und das Problem der demokratischen Repräsentation.

O Nr.68* Politik und Religion in Europa. Mit Beiträgen von Orlando Budelacci und Gabriel N. Toggenburg.

O Nr.69 Chantal Delli, Das Wesen der Unionsbürgerschaft, Überlegungen anhand des Falls Rudy Grzelczyk gegen Centre public d'aide sociale d'Ottignies-Louvain-la-Neuve.

O Nr.70 Federica Sanna, La garantie du droit de grève en Suisse et dans la L'Union européenne.

O Nr.71 Elze Matulionyte, Transatlantic GMO Dispute in the WTO: WIII Europe futher abstain from Frankenstein foods?

ONr.72 Rolf Weder (Hrsg.), Parallelimporte und der Schweizer Pharmamarkt.

O Nr.73 Marc Biedermann, Braucht die EU Lobbying-Gesetze? Annäherungen an eine schwierige Frage.

ONr.74 Hanspeter K. Scheller, Switzerland's Monetary Bilateralism. The episode of 1975.

ONr.75 Bruno Kaufmann/Georg Kreis/Andreas Gross, Direkte Demokratie und europäische Integration. Die Handlungsspielräume der Schweiz.

O Nr.76 Daniel Zimmermann, Die Zwangslizenzierung von Immaterialgüterrechten nach Art. 82 EG. Schutz vor Wettbewerb oder Schutz des Wettbewerbs?

O Nr.77 Laurent Goetschel/Danielle Lalive d'Epinay (Hrsg.), Die Beziehungen zwischen Wirtschaft und Aussenpolitik: Eine natürliche Symbiose?
ISBN-13: 978-3-905751-03-1

ISBN-10: 3-905751-03-8
Europainstitut der Universität Basel, Gellertstrasse 27, $\mathrm{CH}-4020$ Basel, Schweiz, Tel. ++41 (0) 6131797 67, FAX ++41 (0) 613179766

E-mail: europa@unibas.ch, Internet: www.europa.unibas.ch

\section{(c) Europainstitut der Universität Basel 2006}

Alle Rechte vorbehalten. Nachdruck, auch auszugsweise, sowie die Verbreitung auf elektronischem, photomechanischem oder sonstigem Wege bedürfen einer schriftlichen Genehmigung des Europainstituts. 
ISBN-13: 978-3-905751-03-1

ISBN-10: 3-905751-03-8 